\title{
Interaction of a Spark Discharge with W-Cu Electrodes Alloyed by REE
}

\author{
V.Kurochkin and L. Kravchenko \\ Institute for Problems of Material Science, National Academy of Science of Ukraine, 03142, \\ Krzhizhanovskystr.3,Kyiv,Ukraine.E-mail:vkur@ipms.kiev.ua
}

\begin{abstract}
Mathematical simulation and experimental study were used to investigate interaction of a spark discharge in air with W-Cu electrodes alloyed by rare earth elements (REE). Temperature, electron density and concentration of all plasma components, pressure, velocity of fluid jet from the surface of electrodes were obtained in discharge axis at power flux density up to $10^{10} \mathrm{~W} / \mathrm{m}^{2}$. The work also discusses the influence of input power and pressure on effective ionization potential, temperature, electron density and redistribution of energy losses of electrons in inelastic processes. Results demonstrate such additions to be quenching agents, decreasing electron concentration at certain conditions, maximal temperature in current channel and therefore energy flux into material.
\end{abstract}

\section{INTRODUCTION}

W-Cu composition is known as a material widely used for high current electrical contacts and electrodes. It was reported that the addition of an emission-active phase such as $\mathrm{LaB}_{6}$ sufficiently improves the erosion resistance of cathode material $/ 1 /$. Such additions influence the localisation of current channel on the surface of an electrode and decrease energy flux into electrode. Metal vapour erasing as a result of erosion of electrodes has a significant influence on discharge parameters. It increases radiated power and decreases dielectric recovery rate. This results in increasing of extinction time and critical extinction gap length $/ 2 /$. In general metal vapour is assumed to increase electron concentration, taking into account their low ionization potential. But more detailed study reveals the multiparametric nature of the processes. Moreover, in some conditions metal vapour may decrease electron temperature due to the inelastic collisions of electrons and this results in decrease of electron concentration $/ 3 /$. It was shown that metals with low secondary ionization potential and high statistical sums more effectively dissipate energy of electrons than metals having inverse parameters. From this point of view it is especially important to study the influence of REE atoms, known as elements with relatively low secondary ionization potential. The study of interaction of a short impulse discharge with W-Cu (70:30) high temperature materials alloyed as an example by cerium is the subject of this paper.

\section{EXPERIMENTAL DETAILS AND MODEL}

Discharge is generated by the spark generator used in emission spectrometry. Its design allows the energy of an impulse to be obtained independent of dielectric recovery rate of discharger and therefore on the properties of material tested. This is achieved due to the auxiliary discharger that controls discharge energy. Parameters of the generator were as follows:

Capacitor voltage $-12.6 \mathrm{kV}$

Capacitance $\quad-0.02 \mu \mathrm{F}$

Inductance - off

Discharge gap - $1 \mathrm{~mm}$ 
Measurements show that the breakdown stage of such an impulse discharge is approximately $10^{-7} \mathrm{~s}$ at voltage $12 \mathrm{kV}$. The next stage realised the main energy stored in the capacitor and lasted $1-5 \mu \mathrm{s}$ at a voltage of about $120 \mathrm{~V}$ at maximum impulse current $100 \mathrm{~A}$. Average diameter of the current channel is about $50 \mu$ and measured on the polished surface from a single impulse. Such parameters provide discharge power flux up to $10^{10} \mathrm{~W} / \mathrm{m}^{2}$. According to the data reported such discharge emitted metal vapour as a supersonic jet in velocity range $10^{3}-10^{4} \mathrm{~m} / \mathrm{s} / 4 /$. At such conditions diffusion transfer may be neglected because transfer of vapours is determined by the jets. In the present work this parameter is used as an initial approach in the model. Interaction of vapour jets with the surface of opposite electrode and pinch effect result in compressed area. Evaluations show that pressure at above parameters in discharge ax is is about $1 \mathrm{MPa}$.

Parameters of the plasma have been measured by spectrometric methods. The axis of the discharge was projected to coincide with the axis of a spectrometer. This allows the measurement of parameters close to the values existing in the current channel. Location of a discharge axis is unstable mainly due to the instability of the foot of the discharge. To obtain the maximal values of parameters possible, measurements were made with the use of 10 lines of double charged ions WII with excitation energies in a range $5-8 \mathrm{eV}$. Optimal temperatures of such ions lies above $15000 \mathrm{~K}$ and represents processes in the discharge axis /3/. Electron concentration was measured using the Saga equation.

Spark plasma at atmospheric pressure in air has high density so energy exchange between particles is sufficient to establish local thermodynamic equilibrium (LTE). This is validated by estimation of excitation temperature from the slope of the Bolzman plot and electron concentration from the Saga equation. Therefore the difference in temperature of heavy particles and electron temperature may be ignored. Since electrical discharges are inhomogeneous in space and time so all further calculations related to the discharge axis at maximal energy released by capacitor.

The model includes flow continuity conditions for each $\alpha$ component of electrode (Eqn.1), quasi-neutrality condition for plasma (Eqn.2), the equation of state (3), the equation of energy conservation for electrons (4), equations of equilibrium for reactions of dissociation molization for main gas components $\left(\mathrm{AB}=\mathrm{N}_{2}, \mathrm{O}_{2}, \mathrm{NO}\right)$ (5) and Saga equations for all plasma components $(k=$ $\mathrm{N}, \mathrm{O}, \mathrm{NO}$, components of the electrode) (6); equation stated the constant relationship between nucleus of $\mathrm{O}$ and $\mathrm{N}$ in air plasma (7):

$$
\begin{aligned}
& J_{\alpha}=u\left(n_{\alpha}^{0}+n_{\alpha}^{+1}+n_{\alpha}^{+2}\right), \\
& n_{N+}+n_{N O+}+n_{O+}+\Sigma n_{\alpha}^{+1}+2 \Sigma n_{\alpha}^{+2}=n_{e}, \\
& 2 n_{e}+n_{N 2}+n_{N}+n_{O 2}+n_{O}+n_{N O}+\Sigma n_{\alpha}^{0}=P / T, \\
& \Sigma v_{\alpha} E_{\alpha}^{h}=\left(e^{2} E_{0}^{2}\right) / m v_{e}^{s} \\
& n_{A} n_{B} / n_{A B}=K_{A B}(T, \\
& n_{e} n_{k}^{+j} / n_{k}^{j-1}=S_{k}^{j}(T) \\
& 2 n_{N 2}+n_{N+}+n_{N}+n_{N O}+n_{N O+}=3.72 \\
& \left(n_{O 2}+n_{O}+n_{O+}+n_{N O}+n_{N O+}\right) .
\end{aligned}
$$

Here at the right hand side of Eqn.4 the energy of electrons is gained in electric field $E_{0}, v_{e}{ }^{3}$ - summed frequency of collisions; $P$ - pressure; $T$ - temperature in energy units; $J_{\alpha}$ - evaporation rate of a component $\alpha$ with concentration $n_{a} ; u$ - velocity of propagation of vapour jets. Absolute values of evaporation rate of metals $J_{\alpha}$ have been measured previously and lie in the range from $2 * 10^{22} \mathrm{~cm}^{-2} \mathrm{~s}^{-1}$ for $W$ to approximately $5^{*} 10^{24} \mathrm{~cm}^{-2} \mathrm{~s}^{-1}$ for metals with relatively low energy of evaporation $(\mathrm{Ag}, \mathrm{Al}, \mathrm{Mg}$ ) /3/. Since temperatures in the discharge axis are rather high in calculation of $n_{e}$ ionization of ions $\mathrm{Cu}^{+1}, \mathrm{~W}^{+1}$ and $\mathrm{Ce}^{+1}$ were taken into account, i.e. contribution of double charged particles (charge $j=1,2$ in Eqn. 6). Saga functions $S_{k}{ }^{j}(T)$ were calculated with correction of ionization potential by fields of charged particles $/ 5 /$ :

$$
\delta U^{\prime}=e^{2} /\left(T / 8 \pi e^{2} n_{e}\right)^{1 / 2}
$$

Distribution functions of atoms and ions were calculated with use of polynomial approximations $/ 6 /$. 
Equilibrium constants $K_{A B}(T)$ were calculated with use of polynomial approximation for Gibbs potentials $/ 7 /$.

Sum impact frequency is determined from the following equation.

$$
v_{e}^{s}=\Sigma v_{e \alpha}{ }^{t}+\Sigma v_{e \alpha}{ }^{h},
$$

where $v_{e a}{ }^{t}$ and $v_{c a}{ }^{h}$ represent transport and inelastic collision frequency respectively. For Maxwellian distribution of electrons according to velocities dependence $v_{\mathrm{ea}}{ }^{\mathrm{h}}$ is given by the following equation:

$v_{e \alpha}{ }^{h}=\left(8 T_{e} / \pi m_{e}\right)^{1 / 2} n_{\alpha} Q_{e \alpha}{ }^{h}\left(1+2 T_{e} / E_{\alpha}{ }^{h}\right) \exp \left(-E_{\alpha}{ }^{h} / T_{e}\right),(10)$

where $E_{\alpha}{ }^{h}$ - energy of inelastic collisions.

A cross section of ionization has been calculated with use of the Thomson formula /8/:

$$
Q_{e a}{ }^{i}=Z\left(\pi e^{4} / K\right)\left(1 / E^{i}-1 / K\right)
$$

in the energy range $\mathrm{K}=(1.1-2) \mathrm{E}^{\mathrm{i}}$. As concerns of excitation cross section they were approximated by the formula $/ 5 /$ :

$$
\mathrm{Q}_{\mathrm{ea}}{ }^{\mathrm{h}}=(0.00036 \mathrm{~T}-0.1)^{*} 10^{-20}\left[\mathrm{~m}^{2}\right]
$$

This approximation gives quite acceptable results because energy losses are determined by the exponent in formula (10) and variation of concentration within several orders of magnitude rather than by difference in cross sections.

Effective ionization potential is a convenient thermodynamic potential to trace reaction of the plasma on external conditions. In this work we have used a formula obtained previously $/ 9 /$ :

$$
\mathrm{E}_{\mathrm{eff}}=-\underset{\alpha}{\operatorname{Ton}}\left(\sum_{\alpha}\left(\mathrm{g}_{\alpha}{ }^{j} / \mathrm{g}_{\alpha}{ }^{j-1}\right) c_{\alpha} \exp \left(-\mathrm{E}_{\alpha}{ }^{i} / \mathrm{T}\right)\right)
$$

In this formula $\mathrm{g}_{\alpha}{ }^{\mathrm{j}}, \mathrm{g}_{\alpha}{ }^{\mathrm{j}}=$ statistical weights of $\mathrm{j}$ times ionized atom $(j=1,2), c_{\alpha}$ atomic concentration of an $\alpha$ plasma component. Summation is performed over all components contributed into electron concentration. In fact this equation represents a partial Gibbs potential for such element having energy levels equal to the ionization potential of components of the system. In terms of thermodynamics according to such statistical sum ionization potential of each component is its chemical potential.

At the given evaporation rate temperature depends on collision frequency $v_{e}{ }^{5}$ i.e. on plasma composition, and the latter at LTE conditions depends on T. This means that such an object is described by self-consistent sets of equations which were calculated in loops until they reached self-consistent values.

This model is not complete, because it does not include a self-consistent solution for electrical parameters, such as electrical field strength. In order to obtain the necessary parameters we have used combined theoretical and experimental approach. Some parameters such as electrical field strength, diameter of the current channel, discharge duration and derived parameters magnetic field pressure have been found experimentally and used in further calculations as parameters. Electron temperature and electron number density obtained as the result of calculations have been measured in order to check accuracy of the model. Additional details of calculations may be found in the previous work $/ 3 /$. In the present work we would like to concentrate attention on dependence of electron temperature, electron concentration and redistribution of energy losses of electrons in various inelastic processes vs. additions of easily ionized elements, pressure and electric field strength. Such an approach proved to be profitable and reveals some interesting features of interaction impulse current with high temperature materials.

\section{RESULTS AND DISCUSSIONS}

Calculations were made for pressure range $0.5-1.2$ $\mathrm{MPa}$; electrical field strength at maximal current was in a range of $4 \mathrm{E} 4-1 \mathrm{E} 5 \mathrm{~V} / \mathrm{m}$. Figure 1 shows plasma composition against concentration of $\mathrm{Ce}$ in W-Cu composition at constant field strength. Quasi-neutrality conditions are controlled mostly by the parity of $n_{\mathrm{e}}$ and concentration of single charge ions of copper $\mathrm{Cu}^{+1}$ and nitrogen $\mathrm{N}^{+1}$. If, in an arc discharge at a temperature of about $6000 \mathrm{~K}$ the main contributor into electron concentration is the process of ionization of molecules 


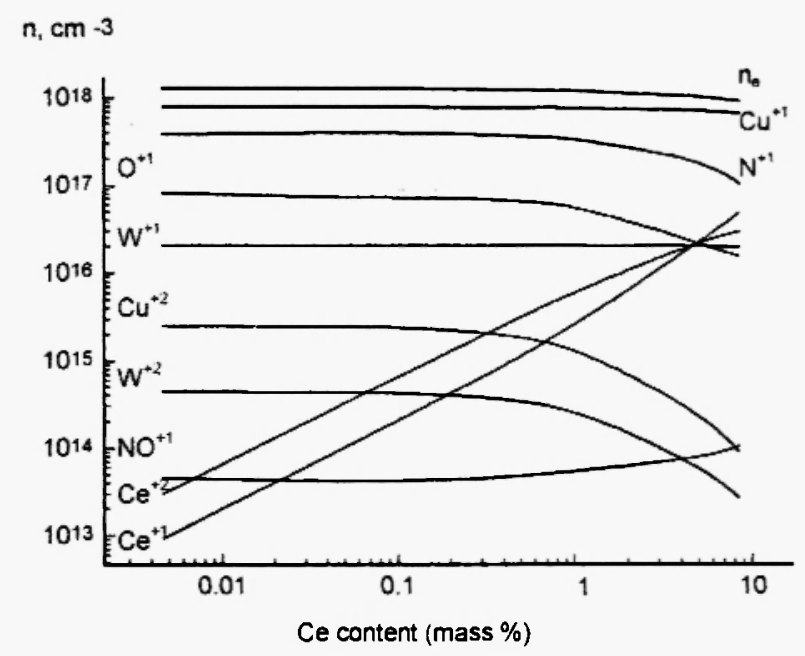

Fig. 1: Concentration of charged particles on discharge axis against addition of $\mathrm{Ce}$ in W-Cu composition at constant power gained by electrons in electric field $E=4 E 4 \mathrm{~V} / \mathrm{m}$

of $\mathrm{NO} / 10 /$ in a spark discharge, these molecules are of secondary importance. These calculations indicate that additions of special quenching molecular gases dissipating energy via electronic, rotational and oscillatory modes for materials working under such extreme conditions as spark discharge are unlikely to cause radically lowered temperature. At such temperatures in discharge axis concentrations of molecular gases are relatively small, compared to those of atoms. Concentration of $\mathrm{NO}^{+}$is four orders of magnitude less than electron concentration and concentrations of atomic gases. Atoms of $\mathrm{Ce}$ have relatively low second ionization potential so at the temperatures above $13000 \mathrm{~K}$ practically all $\mathrm{Ce}$ is in the form of double charged ions, $\mathrm{Ce}^{+2}$. Only at concentrations of cerium above $5 \%$ when temperature became less than $13000 \mathrm{~K}$ do concentrations of $\mathrm{Ce}^{+1}$ exceed those of $\mathrm{Ce}^{+2}$. Calculations show in general rather weak dependence of electron density for concentrations below $0.1-0.5 \%$. This is clearly visible in Fig. 2 in normalized units. At concentration of cerium above $1-8 \% n_{e}$ decreases by $7-30 \%$. Calculations show that addition of $\mathrm{Ce}$ does not increase electron concentration, as may be expected a priori; moreover this results in clearly visible decrease of $n_{e}$. The reason for such behaviour is a decrease in electron temperature

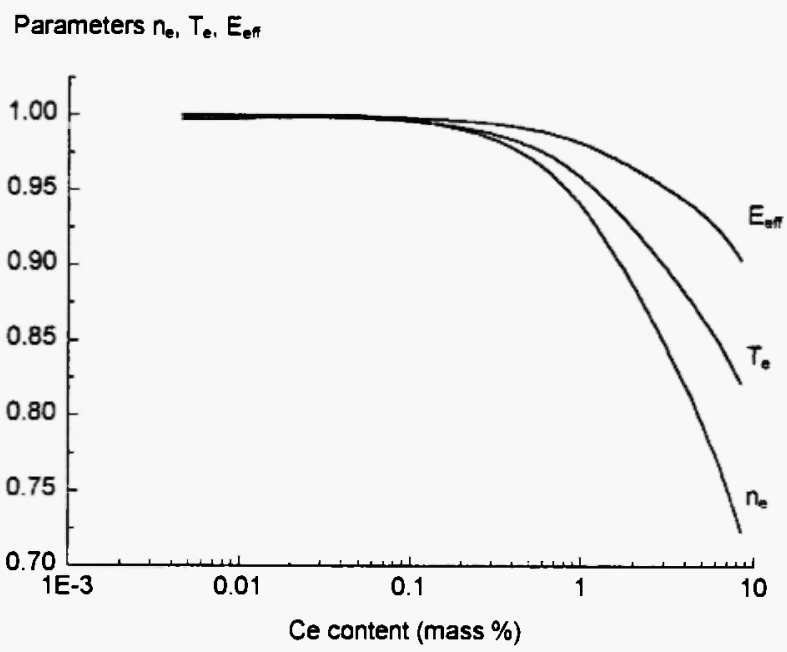

Fig. 2: Normalized values of discharge parameters vs. $\mathrm{Ce}$ concentration in $\mathrm{W}-\mathrm{Cu}$ composition at constant power gained by electrons in electric field $\mathrm{E}=4 \mathrm{E} 4 \mathrm{~V} / \mathrm{m}$. Maximal values are as follows: electron concentration $n_{e \max }=1.3 \mathrm{E} 18$ $\mathrm{cm}^{-3}$; temperature of electrons $T_{\mathrm{e} \max }=15800$ $\mathrm{K}$; Effective ionization potential of the gas mixture $E_{\text {eff } \max }=12.95 \mathrm{eV}$

due to inelastic collisions with excitations of levels. But decrease in $n_{e}$ is not as high as may be expected, taking into account exponential dependence of electron concentration of $T$ (Saga function (6)). The point is that there is a simultaneous decrease in effective ionization potential of the gas mixture $E_{\text {eff }}$ (Fig. 2). The combined effect of these factors results in a relatively small decrease in electron concentration. Additions of cerium also change the dependence of electron temperature of electric field strength i.e. of power gained by electrons in this field. The enhancement of cerium concentration cause temperature to decrease and make dependence weaker (Fig.3). In general such an addition serves as a quenching agent which lowers energy flux into the electrode surface and extinction time.

The next stage in understanding the processes of interaction of electrode materials with plasma give alterations of ambient conditions - pressure and discharge power. Computer-generated solutions are shown in Figs. 4 and 5 for W-Cu material with $5 \% \mathrm{Ce}$. Nontrivial results demonstrate dependencies of temperature and electron concentration of pressure (Fig. 


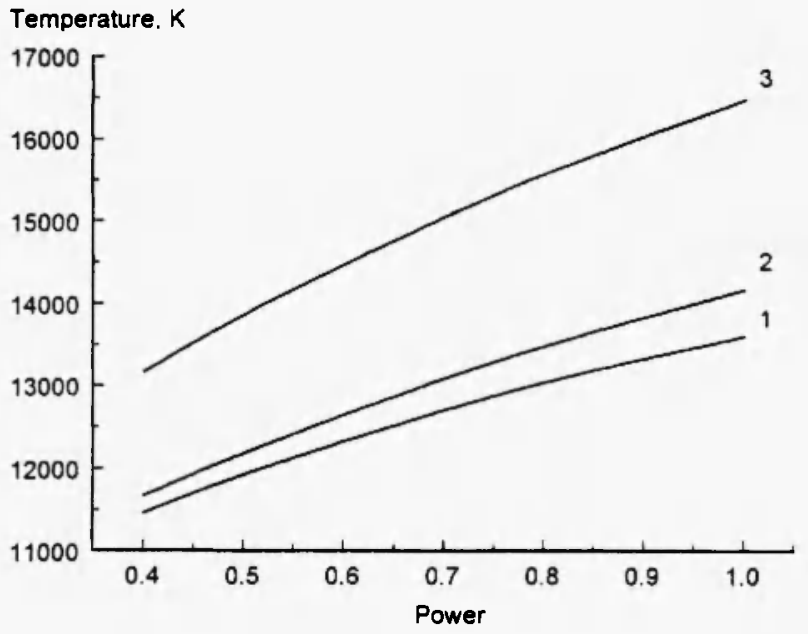

Fig. 3: Relationship between power gained by electrons in electric field and electron temperature on discharge axis for various concentration of cerium in W-Cu composition. Maximal power corresponds to the electric field strength IE5 V/m. 1 - $6 \% \mathrm{Ce} ; 2-4 \% \mathrm{Ce}$; 3 - without addition of $\mathrm{Ce}$.

4(a), 4(b)). Increase in pressure by $200 \%$ at constant low power causes $T$ to decrease only by several percent. In the range of higher powers this dependence is stronger but still not as high as expected, bearing in mind Eqn. 10. The key to these questions lies with dependencies of energy losses in inelastic processes shown in Fig. 5(a), 5(b). Comparison of these curves indicates redistribution of energy losses in various processes. At the low power the main channel of energy dissipation is excitation of levels of metals $\left(\mathrm{P}_{\text {exc.Me }} \cong 90 \%\right)$ whereas the contribution of gas levels $\left(\mathrm{P}_{\text {exc.g. }}\right.$ ) is less than $10 \%$. A process of ionization is responsible for the rest of the energy losses.

Fig. 4: Normalized values of parameters of a spark discharge for W-Cu electrode with $5 \%$ of $\mathrm{Ce}$ against pressure and power gained by electrons in electric field. Maximal value of power calculated for electric field $E=1 E 5 \mathrm{~V} / \mathrm{m}$; maximal pressure $-\mathrm{P}_{\max }=1.2 \mathrm{E} 6 \mathrm{~Pa}$; (a) electron concentration, $\mathrm{n}_{\mathrm{e} \max }=1.3 \mathrm{E} 18 \mathrm{~cm}^{-3}$; (b) - temperature, $T_{\max }=15800 \mathrm{~K}$; (c) - effective ionization potential, $\mathrm{E}_{\mathrm{eff} \max }=12.95 \mathrm{eV}$.

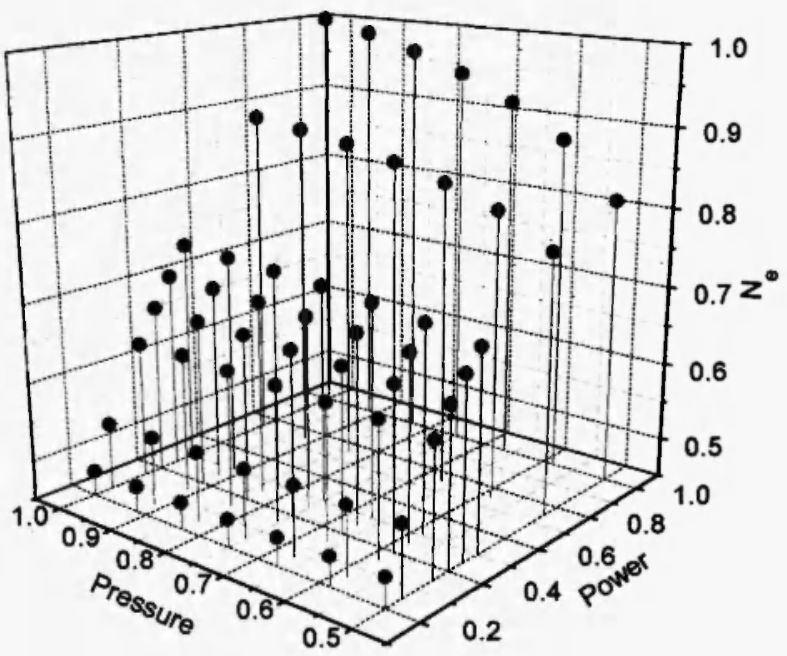

(a)

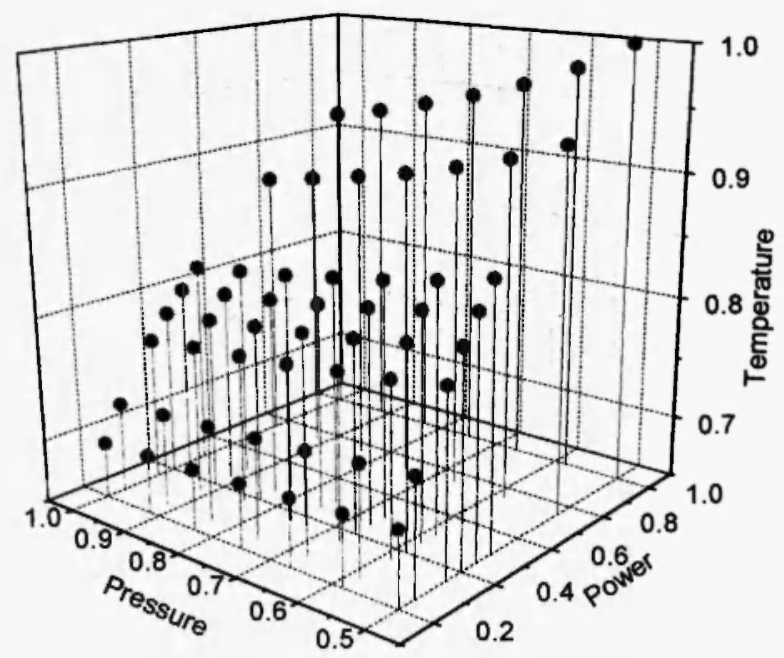

(b)

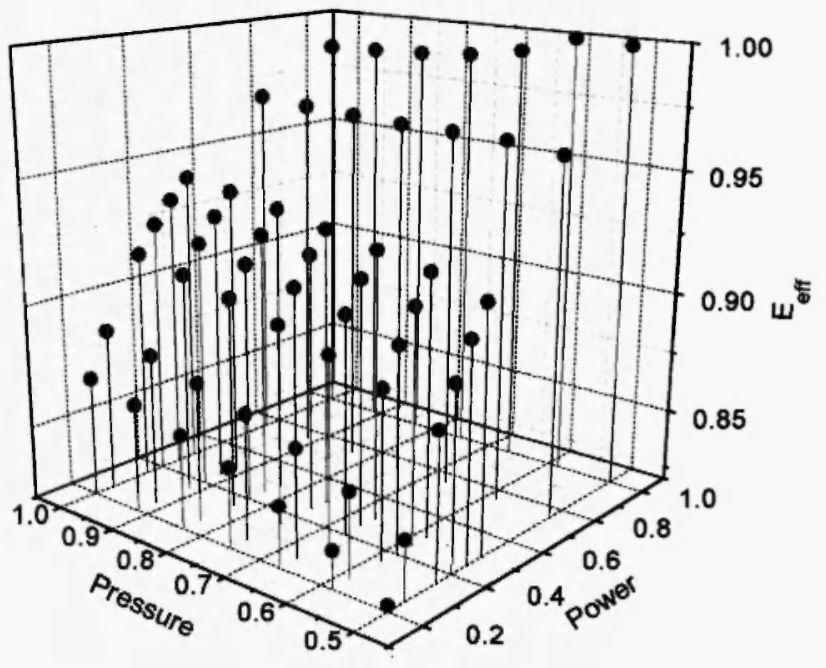

(c) 


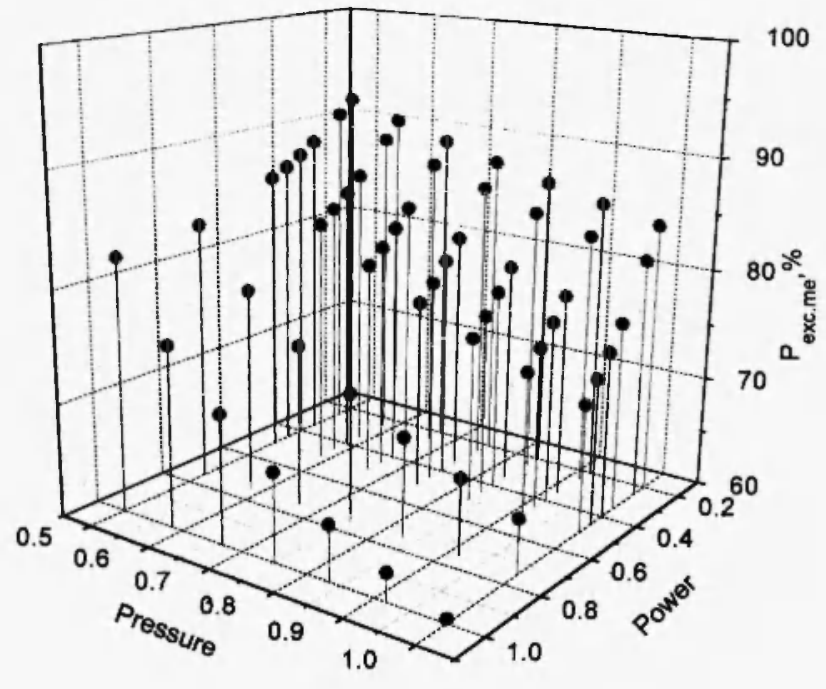

(a)

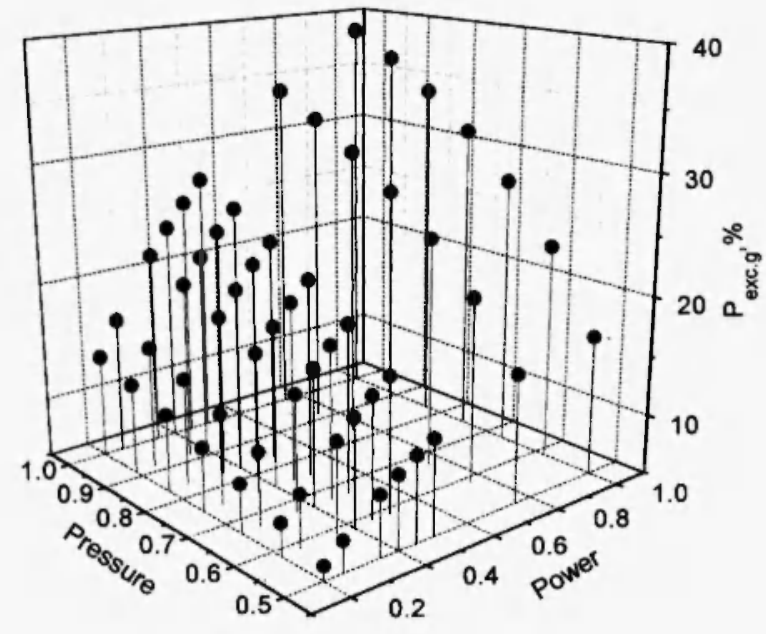

(b)

Fig.5: Relative energy losses of electrons in inelastic processes for W-Cu electrodes with $5 \%$ of $\mathrm{Ce}$ against pressure and power gained by electrons in electric field. Maximal value of electric field $\mathrm{E}=1 \mathrm{ES} \mathrm{V} / \mathrm{m}$; maximal pressure $-\mathrm{P}_{\max }=1.2 \mathrm{E} 6$ $\mathrm{Pa}$; (a) - excitation of metals; (b) - excitation of gases

Increase in pressure results in decreasing of $i_{\text {exc. Me }}$ by $5 \%$ but simultaneously increases $\mathrm{P}_{\text {exc. }}$ by approximately the same value. The same picture but more obvious is seen in high power range. As a result temperature only slightly decreases with pressure. Electron density in current channel (Fig.4(a)) is mainly determined by the temperature. From the Fig. 4(a), 4(b) it is seen that at high power $n_{e}$ increases with pressure whereas $T$ in the same conditions decreases. These results seem to be in contradiction with the Saga equation, but comparison with effective ionization potential $\left(E_{\text {eff }}\right)$ at the above conditions shows that $E_{\text {eff }}$ also decreases (Fig.4(c)). The combined effects of pressure, temperature and changing of $E_{\text {eff }}$ result in rather stable value $n_{e}$ and at high power it even increases.

Increase in electron concentration with temperature is to be expected, but as temperature increases by 20 $30 \%$ electron concentration increases by only $30-50 \%$. This figure is not as high as would be expected if effective ionization potential is constant. Following the Saga equation there must be exponential dependence $n_{e}$ of T. Comparison with Fig.4(c) answers this question. Effective ionization potential also increases with power approximately by the same value as temperature does. Dependence of effective ionization potential of power and pressure in general is very similar to that for temperature (Fig.4(b)). This is quite natural as $E_{e m}$ is a function of temperature (Eqn.13). But in the range of low values of power $E_{\text {eff }}$ increases with pressure whereas temperature slightly decreases. In this range of power the contribution of relatively high gas levels is more significant than the effect of temperature. At high power behaviour of both surfaces is practically the same.

Measurements of $n_{e}$ and $T$ are used as a basic guideline in deciding how correct are the proposed model and results obtained. Measured electron density and temperature within error of calculations and measurements (about $20 \%$ ) agree with calculated values. This indicates correctness of the model and above evaluations of electrical discharge parameters, pressure and velocity of vapour jets. The obtained value of $u \cong$ $10^{4} \mathrm{~m} / \mathrm{s}$ agreed with that previously reported in $/ 4 /$.

Another principal criterion is correspondence of $E_{\text {eff }}$ and other properties of the system with general principles of thermodynamics. Calculations show that changing of ambient conditions - pressure and discharge power produce such a change of $T$ and effective ionization potential as to minimise change of $n_{e}$. In practice this means that an increase in discharge power results in the appearance of new higher levels available for the system and in an increase of dissipation of 
energy. This slows down increase in $\mathrm{T}$ and electron concentration.

The results obtained make it possible to explain, in particular, the weak dependence of extinction gap length of erosion rate of electrodes. Therefore an increase in the order of magnitude of erosion rate results in an increase of only $20 \%$ of gap length $/ 2 /$. However these experiments related to the arc discharge inelastic collisions may result in analogous changes in temperature and electron concentration, as calculated for a spark discharge. Additions of metal vapours result in an increase of radiated power and therefore decrease temperature and electron concentration in a perfect analogy with the Fig. 1 and Fig.2.

Computer-generated solutions show that electron concentration in discharge axis is a complex function of ambient conditions and composition of a material. The proposed approach appears to be useful in obtaining quantitative values of discharge parameters. As indicated in our calculations, the addition of hexaborides of REE may be useful for operating characteristics, not only because of lowering of work function but because of lowering discharge parameters. As a result these factors may decrease erosion rate of electrodes and are expected to increase their life cycle.

\section{REFERENCES}

1. G. Levchenko, R. Luban, P. Verchovodov, V. Kurochkin and V. Stepkin, Surface Physics, Chemistry, Mechanics, 5, 130-133 (1983).

2. A. Gleizes, A.M.A. Amry, A.M. Rahal and S. Vasquie, J. Appl. Phys., 67, 2, 670- 675 (1990).

3. V. Kurochkin, R. Minakova and A. Kresanova, Thermophysics of High Temperatures (Russia) 31, 5, 693-697 (1993).

4. G.V. Butkevich, G.C. Belkin et al., Electrical errosion of high current contacts and electrodes, Moscow, Energy, 1978.

5. V. Engelsht and B. Uryukov, Low Temperature Plasma, Novosibirsk, Nauka, Siberian branch of RAS, 1990.

6. B. Faggeter, G. Heisz and M.W. Blades, Spectrochimica Acta. 42B, 11/12, 1235-1239 (1987).

7. L.V. Gurvich, I.V. Veic and V.A. Medvedev, Thermodynamical Properties of Individual Materials, Moscow, Ed. "Nauka", 1982.

8. V.E. Holant, A.P. Zhilinsky and S.A. Sacharov, Plasma Physics. Moscow, Atomizdat, 1977.

9. V. Kurochkin, Reports of Ukrainian Academy of Science, 3B, 44-47 (1988).

10. V. Kurochkin and L. Kravchenko, J. Analyt. Chem., 44 (11), 1947-1952 (1988). 


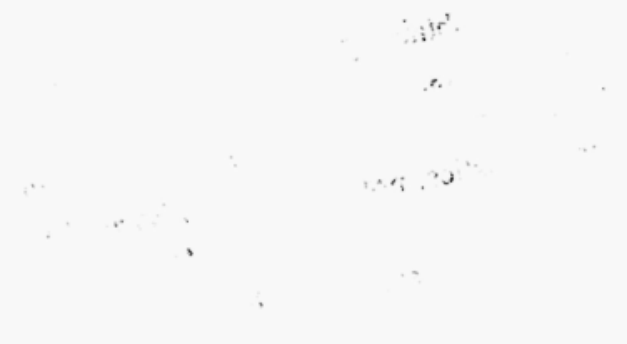

